\title{
Use of rhenium-188 for in vivo imaging and treatment of human cervical cancer cells transfected with lentivirus expressing sodium iodide symporter
}

\author{
MIN ZHANG, SHUO SHI, RUI GUO, YIN MIAO and BIAO LI \\ Department of Nuclear Medicine, Rui Jin Hospital, Shanghai Jiao Tong University, \\ School of Medicine, Shanghai 200025, P.R. China
}

Received April 17, 2016; Accepted August 16, 2016

DOI: $10.3892 /$ or.2016.5034

\begin{abstract}
Although survival rates for cervical cancer have improved, they need further improvement in patients with distant metastases. The sodium iodine symporter (NIS) gene has often been used in cancer therapy and imaging. We examined the therapeutic effects of rhenium-188 $\left({ }^{188} \mathrm{Re}\right)$ in a cervical cancer xenograft model expressing the NIS gene under the control of the tumor-specific human telomerase reverse transcriptase (hTERT) promoter. We constructed two recombinant lentiviral vectors expressing enhanced green fluorescent protein (eGFP) or the NIS gene driven by the hTERT promoter. To determine the tumor-specific transcriptional activity of the hTERT promoter, the eGFP-expressing vector was stably transfected into tumor cells and normal cells. A cervical cancer HeLa cell line stably expressing NIS (HeLaTERTNIS) was created and examined in a similar way. HeLa and HeLa-TERTNIS tumor xenografts were transplanted in
\end{abstract}

Correspondence to: Professor Biao Li, Department of Nuclear Medicine, Rui Jin Hospital, Shanghai Jiao Tong University School of Medicine, 197 Rui Jin 2nd Road, Shanghai 200025, P.R. China E-mail:1b10363@rjh.com.cn

Abbreviations: NIS, sodium iodine symporter; ${ }^{188} \mathrm{Re}$, rhemium-188; hTERT: human telomerase reverse transcriptase; eGFP, enhanced green fluorescent protein; HeLa-TERTNIS, HeLa cell line stably expressing NIS under the control of the hTERT promoter; microSPECT/CT, micro-single photon-emission computed tomography/ computed tomography; ${ }^{99 \mathrm{~m}} \mathrm{Tc}$, technetium- $99 \mathrm{~m} ;{ }^{125} \mathrm{I} /{ }^{131} \mathrm{I}$ : iodide125/131; pLVX-puro, lentiviral vector harboring the puromycin resistance gene; pLVX-hTERT-NIS-puro, lentiviral vector expressing NIS under the control of the hTERT promoter; pLVX-hTERTeGFP-puro, lentiviral vector expressing eGFP under the control of the hTERT promoter; MFI, mean fluorescence intensity; bHBSS, buffered Hanks' balanced salt solution; $\mathrm{NaClO}_{4}$, sodium perchlorate; $\mathrm{SD}$, standard deviation; ROI, region of interest; Conc, radioactivity per volume unit

Key words: sodium iodide symporter, cervical cancer, rhenium-188, lentiviral vector, human telomerase reverse transcriptase nude mice, and in vivo ${ }^{188} \mathrm{Re}$ distribution was measured using micro-SPECT/CT imaging. The therapeutic effects of ${ }^{188} \mathrm{Re}$ were assessed over 21 days on the basis of tumor volume and the immunohistochemical findings of excised tumors. eGFP expression controlled by the hTERT promoter was substantially higher in the tumor cells than normal cells. Quantitative PCR and western blotting confirmed that HeLa-TERTNIS cells expressed high levels of NIS mRNA and protein, respectively. Further, ${ }^{188}$ Re uptake and accumulation were significantly higher in HeLa-TERTNIS cells and xenografts than HeLa cells and xenografts. In vitro and in vivo, ${ }^{188} \mathrm{Re}$ significantly reduced the survival of HeLa-TERTNIS cells and inhibited the growth of HeLa-TERTNIS xenografts, respectively. Immunohistochemical staining showed that HeLa-TERTNIS xenograft tumors expressed higher levels of NIS and caspase-3 and lower levels of Ki-67 than HeLa xenograft tumors. Our findings indicated that hTERT promoter-driven expression of the NIS gene in HeLa cells led to ${ }^{188}$ Re uptake and therapeutic effects. Thus, NIS-based gene therapy and imaging using the hTERT promoter and ${ }^{188} \operatorname{Re}$ may be possible.

\section{Introduction}

Cervical cancer is the fourth most common malignancy and cause of cancer-related death among women worldwide (1). Although the associated mortality rates are generally decreasing because of early detection and effective surgical excision as well as chemoradiotherapy, the 5-year survival rate for women with metastatic cervical cancers is still only $16 \%$ (2). Therefore, novel strategies must be explored to improve the management of metastatic cervical cancer.

Sodium iodide symporter (NIS) is a membrane glycoprotein that mediates the transfer of iodide into thyroid follicular cells (3). NIS gene-mediated uptake of radioisotopes such as technetium-99m $\left({ }^{99 \mathrm{~m}} \mathrm{Tc}\right)$, iodide-125 $\left({ }^{125} \mathrm{I}\right)$, and iodide-131 $\left({ }^{131} \mathrm{I}\right)$ has been widely investigated as a potential imaging and therapeutic method for thyroid and non-thyroid carcinomas (4). However, to protect normal cells from unnecessary radioisotope uptake, NIS expression must be restricted to tumor cells only. Human telomerase reverse transcriptase (hTERT) is an important component of telomerase, which is highly active in most malignant tumors but inactive in normal 
somatic cells (5). Therefore, transfer of NIS genes under the control of the hTERT promoter is an important breakthrough for ensuring tumor-specific uptake of radioisotopes.

Rhenium-188 $\left({ }^{188} \mathrm{Re}\right)$ is a chemical analog of technetium and a useful radioisotope emitting powerful $\beta$-particles that can be channeled for therapy and $\gamma$-rays for imaging. Dosimetry calculations of $\beta$-particles have indicated that ${ }^{188} \mathrm{Re}$-perrhenate can generate a higher irradiation dose with a wider irradiation range than ${ }^{131} \mathrm{I}(6)$. The physical characteristics of ${ }^{188} \mathrm{Re}$ support the notion that this $\beta$-emitter may be a more suitable radioisotope for treatment and imaging of NIS-expressing tumors.

In the present study, we constructed a lentiviral vector to express a functional NIS gene under the control of the hTERT promoter. The potential of NIS as an imaging and therapeutic gene was investigated in vitro and in vivo using a cervical cancer xenograft model.

\section{Materials and methods}

Generation of recombinant lentiviral vector. A lentiviral vector harboring the puromycin resistance gene (pLVX-puro) was purchased from Clontech (Takara, Dalian, China). The pFastBac-hTERT-NIS vector was generated in our laboratory (7). The Lv-EF1 $\alpha$-OCT4-IRES-eGFP vector was kindly provided by the Institute of Molecular Biology, Chinese Academy of Sciences. Polymerase chain reaction (PCR) was used to introduce $C l a \mathrm{I}$ and $X b a \mathrm{I}$ enzyme sites flanking the hTERT-NIS fragment in the pFastBac-hTERT-NIS vector, and the fragment was then cloned into pLVX-puro using the same enzymes, to generate a functional vector with the NIS gene under the control of hTERT promoter (pLVX-hTERTNIS-puro). Similarly, the eGFP gene was amplified from Lv-EF1 $\alpha$-OCT4-IRES-eGFP by PCR, digested with BamHI and $X b a \mathrm{I}$, and cloned into the BamHI and $\mathrm{XbaI}$ sites of pLVX-hTERT-NIS-puro to generate a vector harboring the eGFP gene under the control of the hTERT promoter (pLVXhTERT-eGFP-puro).

The HEK293T cell line (Cell Bank of the Chinese Academy of Science, Shanghai, China) was cultured in RPMI-1640 medium supplemented with $10 \%$ fetal bovine serum and $1 \%$ penicillin/streptomycin.

Lentivirus particles were generated by co-transfection of HEK293T cells with pLVX-hTERT-NIS-puro or pLVXhTERT-eGFP-puro and the three packaging plasmids pRsv-REV, pMDIg-pRRE, and pMD2G (Biovector Science Lab, Shanghai, China). The viral particles were harvested by collecting the cell culture medium at $48 \mathrm{~h}$ post-transfection; the supernatants were filtered through filters of pore size $0.45 \mu \mathrm{m}$ and centrifuged at $10,000 \mathrm{x} \mathrm{g}$ for $15 \mathrm{~min}$, and the resulting pellet was resuspended in $100 \mu \mathrm{l}$ culture medium.

Cell lines and cell cultures. Human cervical cancer HeLa cells, human anaplastic thyroid cancer C643 cells, human fibroblast MRC-5 cells (Cell Bank of the Chinese Academy of Science, Shanghai, China) and human glioma U87 cells (American Type Culture Collection, Manassas, VA, USA) were maintained in Dulbecco's modified Eagle's medium. Human proximal tubule epithelial HK-2 cells (Shanghai Cell Bank of the Chinese Academy of Science) were cultured in keratinocyte serumfree medium. Follicular thyroid carcinoma FTC-133 cells
(European Collection of Animal Cell Cultures, Salisbury, UK) were cultured in Dulbecco's modified Eagle's medium:Ham's F12 (1:1) medium. All media except keratinocyte serum-free medium were supplemented with $10 \%$ fetal bovine serum and $1 \%$ penicillin/streptomycin. All cell lines were incubated in a $5 \% \mathrm{CO}_{2}$ atmosphere at $37^{\circ} \mathrm{C}$.

The cell lines were infected with pLVX-hTERT-eGFPpuro at a multiplicity of infection of 100 for $24 \mathrm{~h}$. To select cells stably transfected with pLVX-hTERT-eGFP-puro, $0.5-1.0 \mu \mathrm{g} / \mathrm{ml}$ puromycin (Sigma, Sydney, Australia) was added to the medium for four days. HeLa cells stably transfected with pLVX-hTERT-NIS-puro (HeLa-TERTNIS) were obtained using a similar selection method.

Fluorescence intensity measurement. All tumor or normal cell lines stably transfected with pLVX-hTERT-eGFP-puro were seeded into 6-well plates at a density of $2 \times 10^{5}$ cells/well. eGFP expression under the control of the hTERT promoter was quantitatively analyzed using a flow cytometry (BD Bioscience, San Jose, CA, USA) in all cell lines. The mean fluorescence intensity (MFI) of $3 \times 10^{4}$ cells of each cell line was determined by subtracting the basal MFI of the same uninfected cell line.

Quantitative real-time polymerase chain reaction. Total RNA samples from HeLa and HeLa-TERTNIS cells were extracted using the RNeasy Mini kit and reverse-transcribed into cDNA using the Superscript RT kit (Invitrogen, Carlsbad, CA, USA). Quantitative real-time PCR was performed using the SYBR Premix Ex Taq kit (Takara). The NIS gene was amplified using the forward and reverse primers 5'-GTACATTGTAGCC ACGATGCTGTA-3' and 5'-CCGTGTAGAAGGTGCAG ATAATTC-3', respectively. Additionally, GAPDH was co-amplified using the primers 5'-GTCAAGCTCATTTCC TGGTATGAC-3' (forward) and 5'-CTCTCTCTTCCT CTTGTGCTCTTG-3' (reverse). The cycling conditions were $95^{\circ} \mathrm{C}$ for $10 \mathrm{sec}, 40$ cycles at $95^{\circ} \mathrm{C}$ for $5 \mathrm{sec}$ and $60^{\circ} \mathrm{C}$ for $31 \mathrm{sec}$, and one cycle of $95^{\circ} \mathrm{C}$ for $15 \mathrm{sec}, 60^{\circ} \mathrm{C}$ for $1 \mathrm{~min}$, and $95^{\circ} \mathrm{C}$ for $15 \mathrm{sec}$. According to the manufacturer's protocol, NIS expression levels were normalized to those of the GAPDH endogenous reference using the formula: F-value $=2^{-\Delta \Delta \mathrm{Ct}}(8)$. Quantitative real-time PCR was repeated three times, and the mean values were obtained for each specimen.

Western blotting. Lysates of HeLa and HeLa-TERTNIS cells were prepared using standard methods. Western blot analysis was then performed using mouse anti-human NIS (1:500; Thermo Scientific, Fremont, CA, USA) or mouse anti-human GAPDH (1:10000; Abgent, Suzhou, China) antibody in Trisbuffered saline/Tween-20 with overnight incubation at $4^{\circ} \mathrm{C}$, followed by incubation with peroxidase-conjugated goat antimouse IgG (1:2500; Santa Cruz Biotechnology, Santa Cruz, CA, USA) for $1 \mathrm{~h}$ at room temperature. Immunodetection was performed using ECL Western Blot Detection kit (Pierce, Waltman, MA, USA).

In vitro ${ }^{188}$ Re uptake studies. HeLa and HeLa-TERTNIS cells $\left(1 \times 10^{5}\right)$ were seeded in 24-well plates and cultured for $24 \mathrm{~h}$. After subsequent washing with buffered Hanks' balanced salt solution (bHBSS), the cells were incubated for $10 \mathrm{~min}$ to $24 \mathrm{~h}$ at $37^{\circ} \mathrm{C}$ with $500 \mu \mathrm{l} \mathrm{bHBSS}$ containing $10 \mu \mathrm{mol} / \mathrm{l}$ sodium iodide 

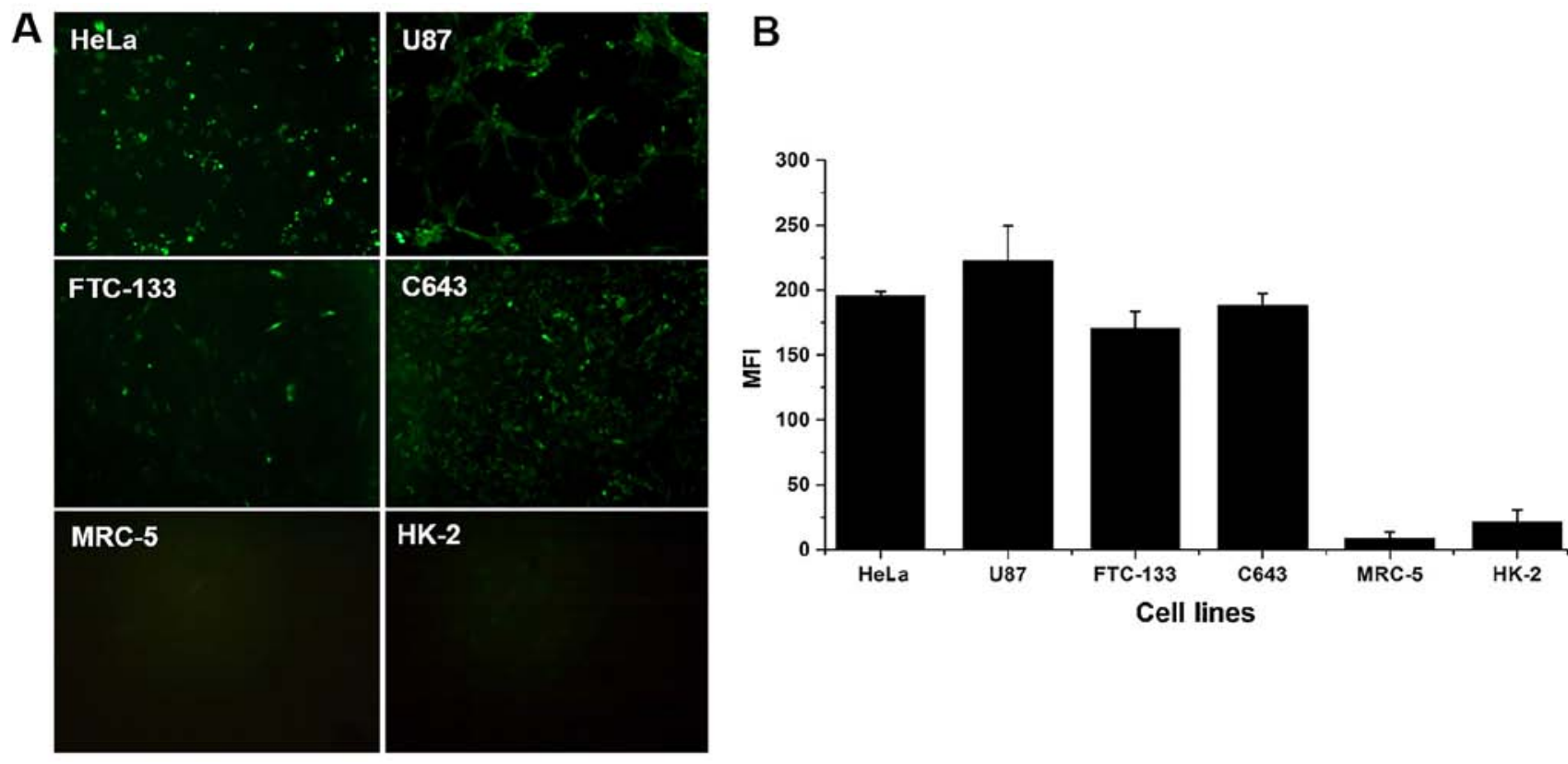

Figure 1. Expression of eGFP under the control of the hTERT promoter in tumor and normal cells. (A) Expressions of eGFP was apparent in all four tumor cell lines (HeLa, U87, FTC-133, and C643) but was almost invisible in normal cell lines (MRC-5 and HK-2) stably transfected with pLVX-hTERT-eGFP-puro. (B) The mean fluorescence intensity (MFI) in the four tumor cell lines was significantly higher than that in the two normal cell lines $(\mathrm{P}<0.01)$.

and $37 \mathrm{kBq}{ }^{188} \mathrm{Re}$ in the form of $\mathrm{Na}^{188} \mathrm{ReO}_{4}$ (Xinke, Shanghai, China). HeLa-TERTNIS cells in the inhibition group were treated with $300 \mu \mathrm{mol} / 1$ sodium perchlorate $\left(\mathrm{NaClO}_{4}\right)$. At various points of incubation, the cells were washed twice with ice-cold bHBSS and lysed with $1 \mathrm{~mol} / \mathrm{l}$ sodium hydroxide. The radioactivity (counts per minute, CPM) was measured using a $\gamma$-counter (Rihuan, Shanghai, China). All experiments were performed in triplicate.

In vitro clonogenic assay. HeLa and HeLa-TERTNIS cells were plated in $10-\mathrm{cm}$ culture dishes $\left(5 \times 10^{6} \mathrm{cells} / \mathrm{dish}\right)$, and $3.7 \mathrm{MBq}{ }^{188} \mathrm{Re}$ in bHBSS was added. After $8 \mathrm{~h}$, the cells were washed three times with bHBSS and then trypsinized. Thousand cells were plated into each well of 6-well plates. On day 7, the cells were stained with $1 \mathrm{ml}$ of crystal violet staining solution (Beyotime Institute of Biotechnology, Shanghai, China) for $10 \mathrm{~min}$, and colonies containing more than 50 cells were counted by observation under a microscope (Olympus, Tokyo, Japan). The survival rate of each group was expressed as the percentage of colonies to the number of colonies formed by HeLa cells not treated with ${ }^{188} \mathrm{Re}$. Data are represented as means \pm standard deviation.

Establishment of xenograft tumors in nude mice. Female BALB/c nude mice aged 4 weeks (Shanghai Slaccas Experiment Animal Corp., Shanghai, China) were s.c. injected with $5 \times 10^{6} \mathrm{HeLa}$ cells in the left thigh and $5 \times 10^{6}$ HeLa-TERTNIS cells in the right thigh. This study protocol was approved by the institutional review board and the experimental animal center of Rui Jin Hospital affiliated to Shanghai Jiao Tong University School of Medicine.

Micro-single photon-emission computed tomography/ computed tomography imaging. Three mice bearing both HeLa and HeLa-TERTNIS tumors were i.v. injected with
$37 \mathrm{MBq}$ of ${ }^{188} \mathrm{Re}$ for micro-single photon-emission computed tomography/computed tomography (micro-SPECT/CT) imaging. The mice was anesthetized by isoflurane inhalation, placed in the spread-prone position, and scanned using a small animal micro-SPECT/CT scanner (Bioscan, Washington, DC, USA) at $0.5,2,4,6$, and $24 \mathrm{~h}$ after injection of the radioisotope. CT images were acquired, after which whole-body SPECT images (10 sec/frame for systematic scans) were obtained. Regions of interest (ROIs) were drawn in the visible tumors and organs including the stomach, lung, liver, intestine, muscle, thyroid, bladder, and heart at various time points. The radioactivity per volume unit (Conc; $\mu \mathrm{Ci} / \mathrm{mm}^{3}$ ) in the ROIs was measured using InVivoScope 1.44 software (Bioscan). To compare in vivo distribution and kinetics of ${ }^{188} \mathrm{Re}$ with those of radioiodide, a micro-SPECT/CT imaging study with $37 \mathrm{MBq}$ ${ }^{125} \mathrm{I}$ was performed 1 week after ${ }^{188} \mathrm{Re}$ imaging in the same mice. ${ }^{125} \mathrm{I}$ was used in this study instead of ${ }^{131} \mathrm{I}$, since both share pharmacokinetic properties but it is easier to quantify energy emitted by ${ }^{125} \mathrm{I}$.

In vivo ${ }^{188}$ Re therapy. For ${ }^{188} \mathrm{Re}$ therapy, 10 mice bearing both HeLa and HeLa-TERTNIS tumors were divided into two groups: one group received $37 \mathrm{MBq}$ of ${ }^{188} \mathrm{Re}$ while the other received saline solution injected via the tail vein on day 0 and the same volume on day 7. Tumor size was measured every 3 days after injection and monitored for 21 days using calipers; tumor volume was calculated using the formula: volume $\left(\mathrm{mm}^{3}\right)=\left(\right.$ length $\mathrm{x}$ width $\left.{ }^{2}\right) / 2$.

Immunohistochemical analysis. At the end of the therapy experiments, the animals were sacrificed by cervical vertebra dislocation, and the tumors were removed and immunohistochemically analyzed using rabbit anti-human NIS antibody (1:50; Proteintech Group, Chicago, IL, USA), rabbit antihuman caspase-3 antibody (1:30; Epitomics, Burlingame, CA, 
A

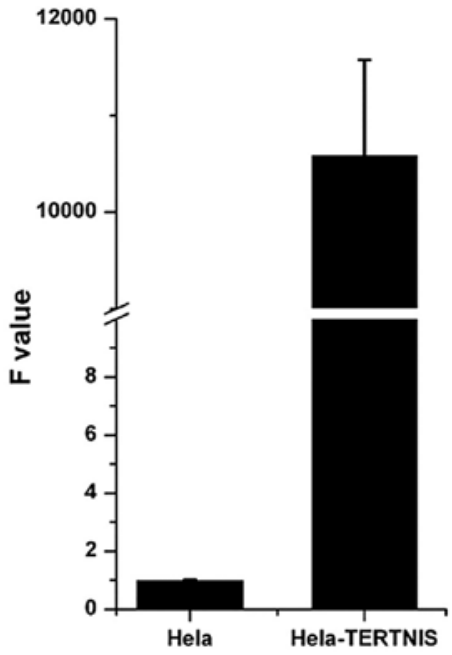

B

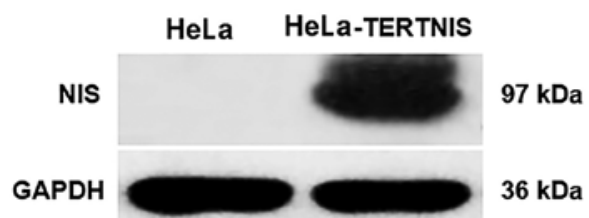

Figure 2. Expression of NIS in HeLa-TERTNIS cells. (A) NIS mRNA expression detected by quantitative real-time PCR and normalized to the GAPDH endogenous reference in HeLa-TERTNIS cells was much higher than that in HeLa cells. (B) NIS protein expression ( $97 \mathrm{kDa})$ was detected in HeLa-TERTNIS cells but not in HeLa cells by western blotting. GAPDH ( 36 kDa) was used as an internal control.

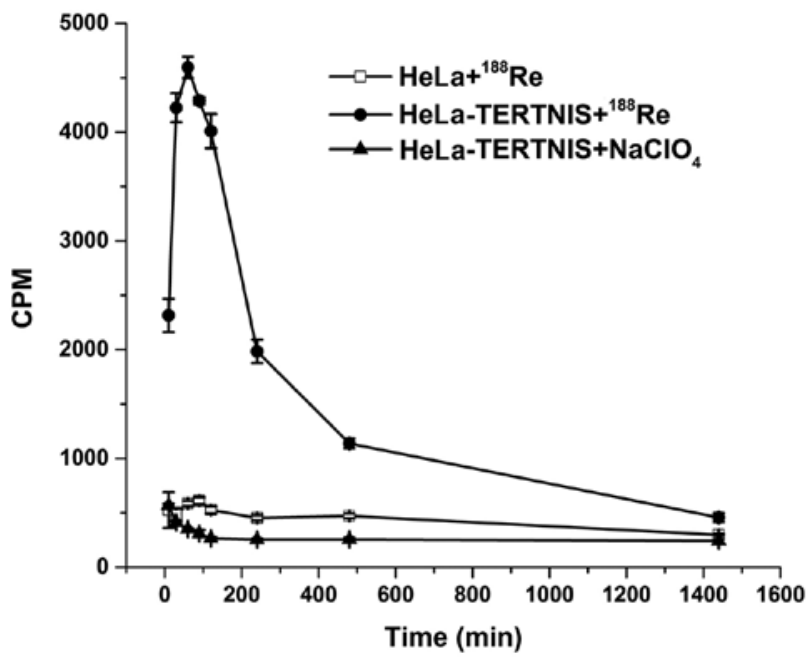

Figure 3. In vitro ${ }^{188} \mathrm{Re}$ uptake assay. The ${ }^{188} \mathrm{Re}$ uptake in HeLa-TERTNIS cells reached a peak after 60 min of incubation, followed by a decline, and was completely blocked by $\mathrm{NaClO}_{4}$. No functional ${ }^{188} \mathrm{Re}$ uptake was observed in HeLa cells. CPM, counts per minute.

USA), and rabbit anti-human Ki67 antibody (1:200; Thermo Scientific). The findings were semi-quantitatively analyzed using Image Pro Plus software (Media Cybernetics, Rockville, MD, USA). For every section, the integral optical density of each visual field was calculated. Data are represented as means \pm SD.

Statistical analysis. Data were analyzed using SPSS 19.0 software (SPSS Inc., Chicago, IL, USA). Each experiment was carried out in triplicate, and results are presented as means \pm SD. Experimental groups were compared by analysis of variance. $\mathrm{P}<0.05$ was considered statistically significant.

\section{Results}

Fluorescence expression of eGFP under the control of the hTERT promoter in tumor and normal cells. To confirm that

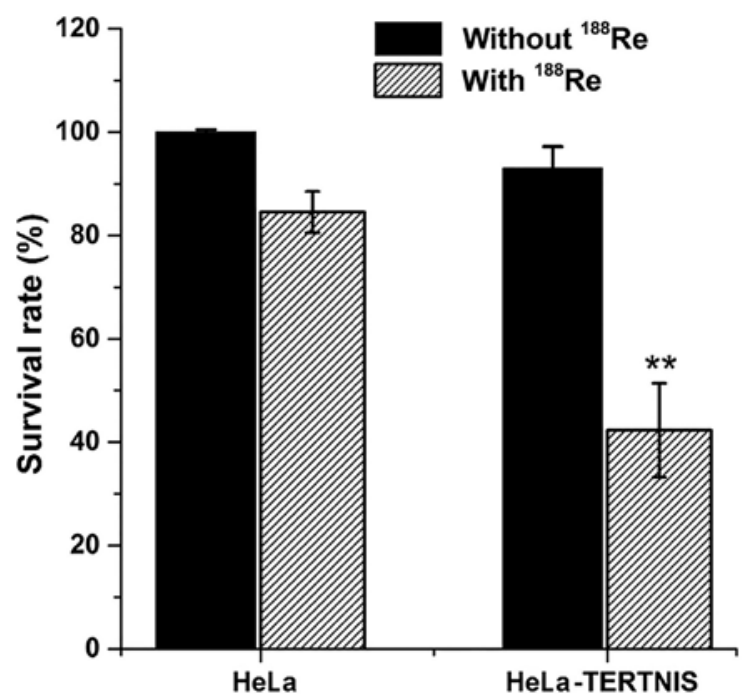

Figure 4. Cytotoxic effect of ${ }^{188} \mathrm{Re}$ in vitro. The survival rate of HeLaTERTNIS cells treated with ${ }^{188} \mathrm{Re}$ was markedly lower than that of HeLa cells treated with ${ }^{188}$ Re and HeLa-TERTNIS cells not treated with ${ }^{188}$ Re. ${ }^{* * *} \mathrm{P}<0.01$.

the transcriptional activity of the hTERT promoter is higher in telomerase-positive tumor cells than in normal cells, eGFP was expressed under the control of the hTERT promoter (Fig. 1A) and quantitatively analyzed (Fig. 1B) in four tumor cell lines (HeLa, U87, FTC-133, and C643) and two normal cell lines (MRC-5 and HK-2) stably transfected with pLVXhTERT-eGFP-puro. The MFI of eGFP in all tumor cells was approximately 10 - to 20 -fold higher than that in the two normal cell lines $(\mathrm{P}<0.01)$.

Stable expression of NIS in HeLa-TERTNIS cells. Quantitative real-time PCR (Fig. 2A) and western blotting (Fig. 2B) confirmed that HeLa-TERTNIS cells expressed high levels of NIS mRNA and protein $(\sim 97 \mathrm{kDa})$, respectively.

In vitro ${ }^{188}$ Re uptake. The functional activity of the NIS protein is shown by its cellular uptake of radioisotopes such as 


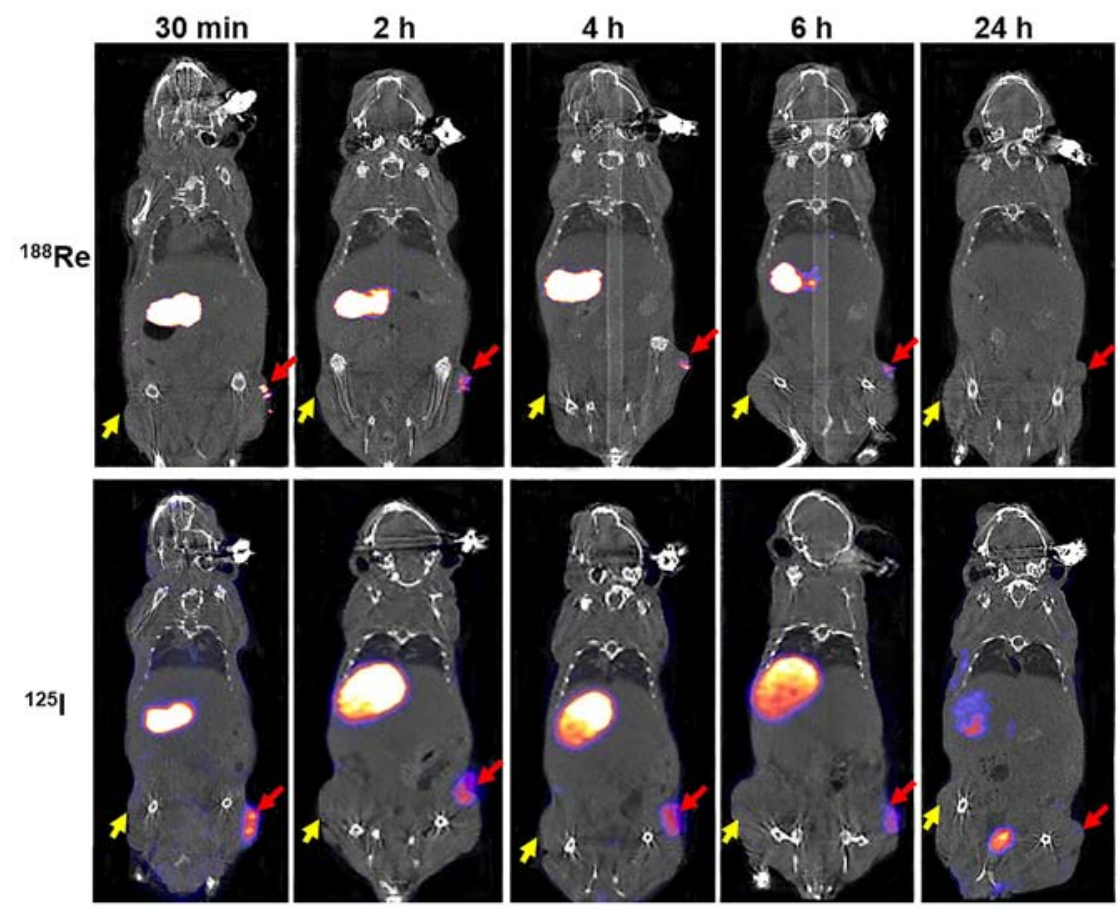

Figure 5. Dynamic ${ }^{188} \mathrm{Re}$ and ${ }^{125}$ I SPECT/CT imaging in vivo. Micro-SPECT/CT imaging of mice bearing both HeLa tumors (yellow arrow) and HeLaTERTNIS tumors (red arrow) at $0.5,2,4,6$, and $24 \mathrm{~h}$ after injection of $37 \mathrm{MBq}{ }^{188} \mathrm{Re}$ or ${ }^{125} \mathrm{I}$.

${ }^{99 \mathrm{~m}} \mathrm{Tc},{ }^{125} \mathrm{I},{ }^{131} \mathrm{I}$, and ${ }^{188} \mathrm{Re}$. As shown in Fig. 3, the ${ }^{188} \mathrm{Re}$ uptake in HeLa-TERTNIS cells rapidly reached a peak (approximately 8-fold higher than that in HeLa cells) after 60 min of incubation, followed by a decline (half-life $\approx 158.4 \mathrm{~min}$ ). No functional ${ }^{188} \mathrm{Re}$ uptake was observed in HeLa cells. Further, the ${ }^{188}$ Re uptake in HeLa-TERTNIS cells was completely blocked by $\mathrm{NaClO}_{4}$.

In vitro clonogenic assay. In vitro clonogenic assays were performed to determine the effect of ${ }^{188} \mathrm{Re}$ in HeLa-TERTNIS and HeLa cells (Fig. 4). The survival rate of HeLa-TERTNIS cells treated with ${ }^{188}$ Re markedly decreased to $42.3 \%$ compared to the $84.5 \%$ survival rate of HeLa cells treated with ${ }^{188} \mathrm{Re}$ $(\mathrm{P}<0.01)$ and $93.0 \%$ survival rate of HeLa-TERTNIS cells not treated with ${ }^{188} \mathrm{Re}(\mathrm{P}<0.01)$. These findings indicating that ${ }^{188} \mathrm{Re}$ emitting $\beta$-particles had a significant cytotoxic effect in HeLa-TERTNIS cells.

In vivo imaging and biodistribution of ${ }^{188}$ Re in mice bearing HeL $a$ and HeLa-TERTNIS xenografts. ${ }^{188}$ Re uptake was clearly observed in HeLa-TERTNIS tumors from 0.5 to $6 \mathrm{~h}$ after injection but was not visible $24 \mathrm{~h}$ after injection. In contrast, ${ }^{188} \mathrm{Re}$ accumulation was not observed in HeLa tumors at any time point after injection (Fig. 5). ${ }^{188} \mathrm{Re}$ accumulation in HeLa-TERTNIS tumors reached the highest Conc value at $0.5 \mathrm{~h}$ after injection, which was significantly (7.6-fold) higher than the corresponding value for HeLa tumors. Additionally, compared to ${ }^{125}$ I uptake, ${ }^{188} \mathrm{Re}$ uptake decreased more rapidly from $0.5 \mathrm{~h}$ onward and reached a substantially lower level at $24 \mathrm{~h}$ (Fig. 6A). Both ${ }^{188}$ Re and ${ }^{125}$ I showed intense accumulation in the thyroid and stomach, which express endogenous NIS, as well as in the bladder, because of renal elimination. However, ${ }^{188} \mathrm{Re}$ cleared at a faster rate than ${ }^{125} \mathrm{I}$ from the thyroid and stomach. The Conc values of both ${ }^{188} \mathrm{Re}$ (Fig. 6B) and
${ }^{125}$ I (Fig. 6C) in the heart, lung, liver, muscle, and intestine remained relatively low compared to thyroid, stomach and bladder at all time points.

Therapeutic effects of ${ }^{188}$ Re in HeLa xenograft tumors expressing NIS. Therapy with $37 \mathrm{MBq}{ }^{188} \mathrm{Re}$ was initiated when the tumors reached $3-5 \mathrm{~mm}$ in diameter. Seven days after the first ${ }^{188} \mathrm{Re}$ injection, the mice were reinjected with $37 \mathrm{MBq}$ ${ }^{188} \mathrm{Re}$ or saline. The growth of HeLa-TERTNIS tumors was significantly inhibited from days 12 to 21 compared to other tumors of the control groups, including HeLa tumors treated with ${ }^{188} \mathrm{Re}$ and HeLa-TERTNIS and HeLa tumors treated with saline $(\mathrm{P}<0.05)$. No significant differences in tumor volume were noted among the three control groups (Fig. 7).

Fig. 8A shows the results of immunohistochemical staining of the tumor xenografts, while Fig. 8B shows the results of quantitative analysis of staining intensity (Fig. 8B). The NIS protein was strongly expressed in HeLa-TERTNIS xenografts treated with ${ }^{188} \mathrm{Re}$ or saline but not in HeLa xenografts with or without ${ }^{188} \mathrm{Re}$ treatment $(\mathrm{P}<0.01)$. Caspase-3 protein expression was significantly higher and Ki67 protein expression was significantly lower in HeLa-TERTNIS xenografts treated with ${ }^{188} \mathrm{Re}$ than HeLa xenografts treated with ${ }^{188} \mathrm{Re}$ HeLa and HeLaTERTNIS xenografts treated with saline $(\mathrm{P}<0.01)$.

\section{Discussion}

Novel strategies to improve the efficacy of cervical cancer treatment are urgently needed since the survival rate of patients with metastatic cervical cancer remains low even after they receive conventional systematic therapy. NIS gene therapy is a promising research area, whereby various radioisotopes can be delivered in concentrated fashion into non-thyroid cancer cell lines, and it has been successfully applied in gliomas, 

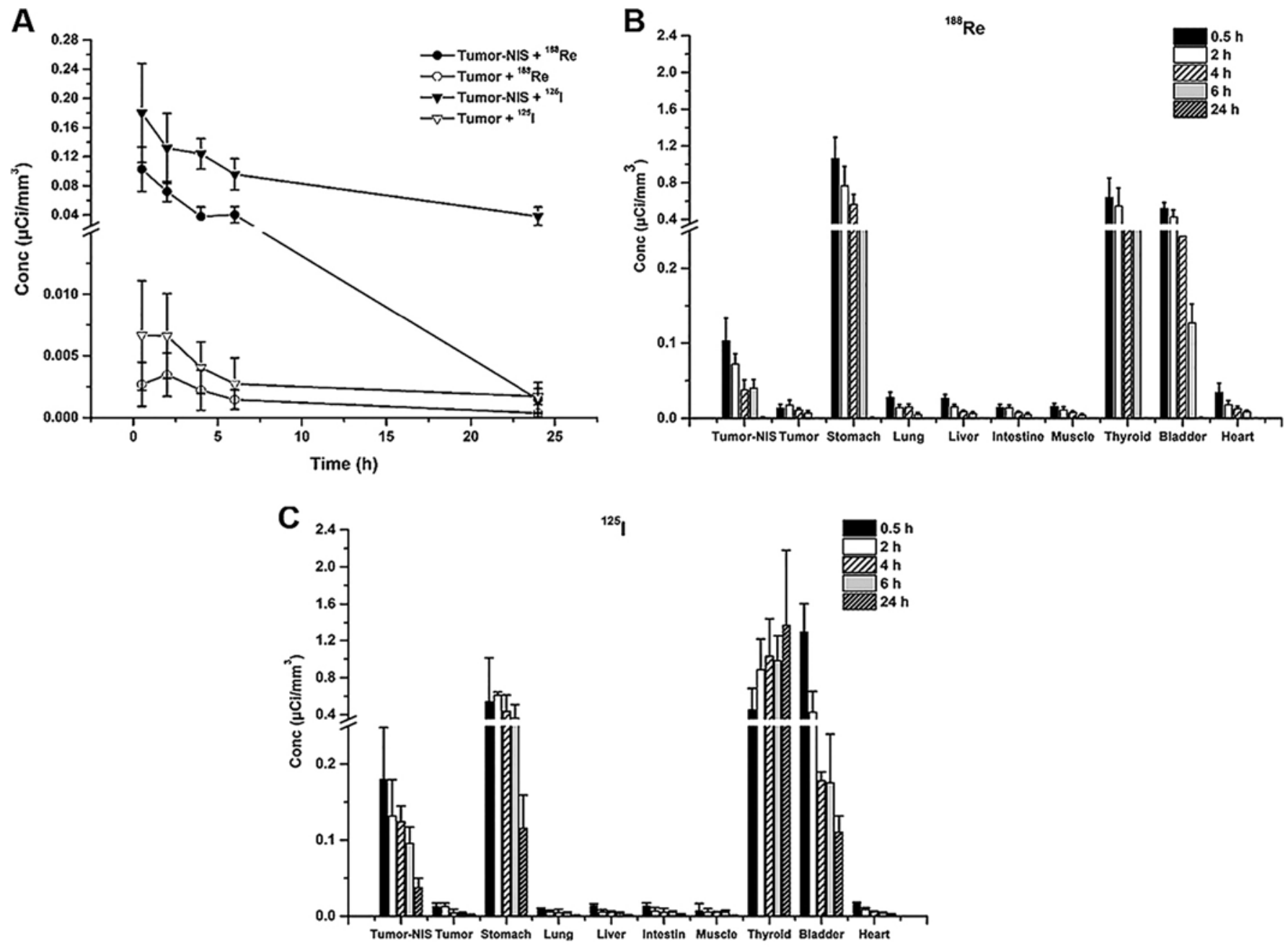

Figure 6. Quantitative analysis of ${ }^{188} \mathrm{Re}$ and ${ }^{125} \mathrm{I}$ uptake in vivo. (A) Biokinetics of ${ }^{188} \mathrm{Re}$ and ${ }^{125} \mathrm{I}$ uptake in the HeLa and HeLa-TERTNIS xenograft tumors. Values of xenograft tumors and various organs at $0.5,2,4,6$, and $24 \mathrm{~h}$ after injection of ${ }^{188} \mathrm{Re}(\mathrm{B})$ or ${ }^{125} \mathrm{I}(\mathrm{C})$. Conc, radioactivity per volume unit.

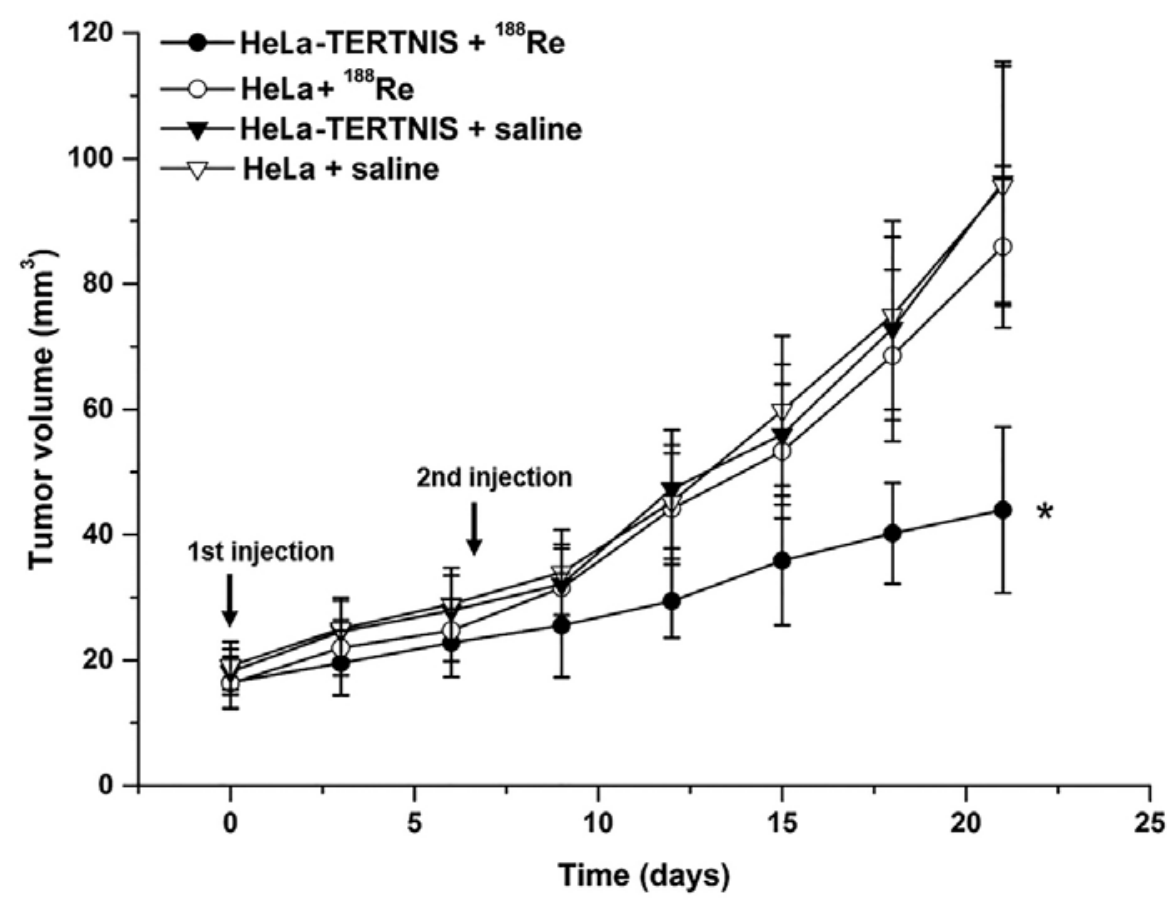

Figure 7. Therapeutic effects of ${ }^{188}$ Re in HeLa-TERTNIS xenografts. Changes in tumor volume over 21 days after injection of ${ }^{188}$ Re or saline. Data are presented as means $\pm \mathrm{SD} .{ }^{*} \mathrm{P}<0.05$. 
A

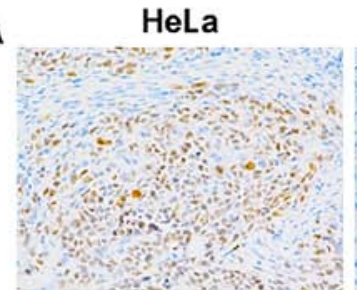

$\mathrm{HeLa}+{ }^{188} \mathrm{Re}$
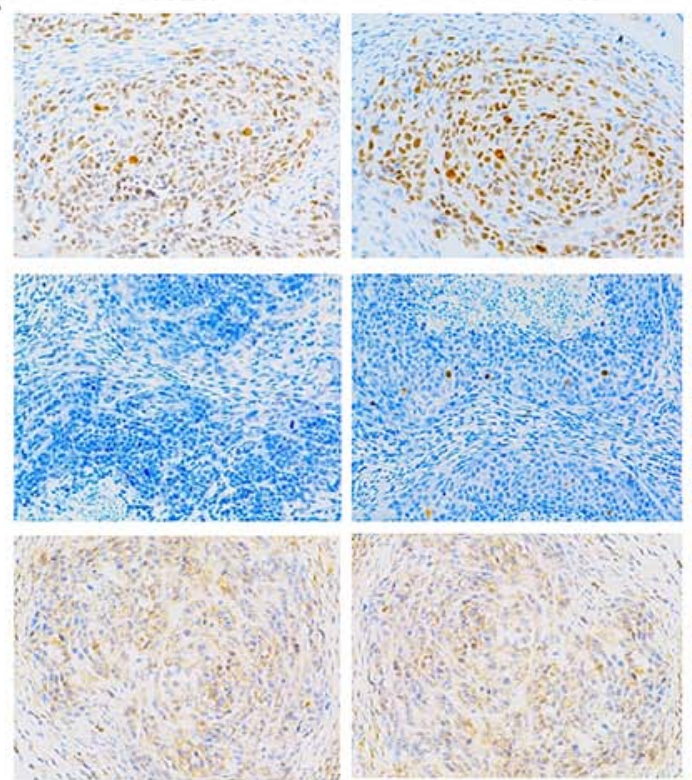

B

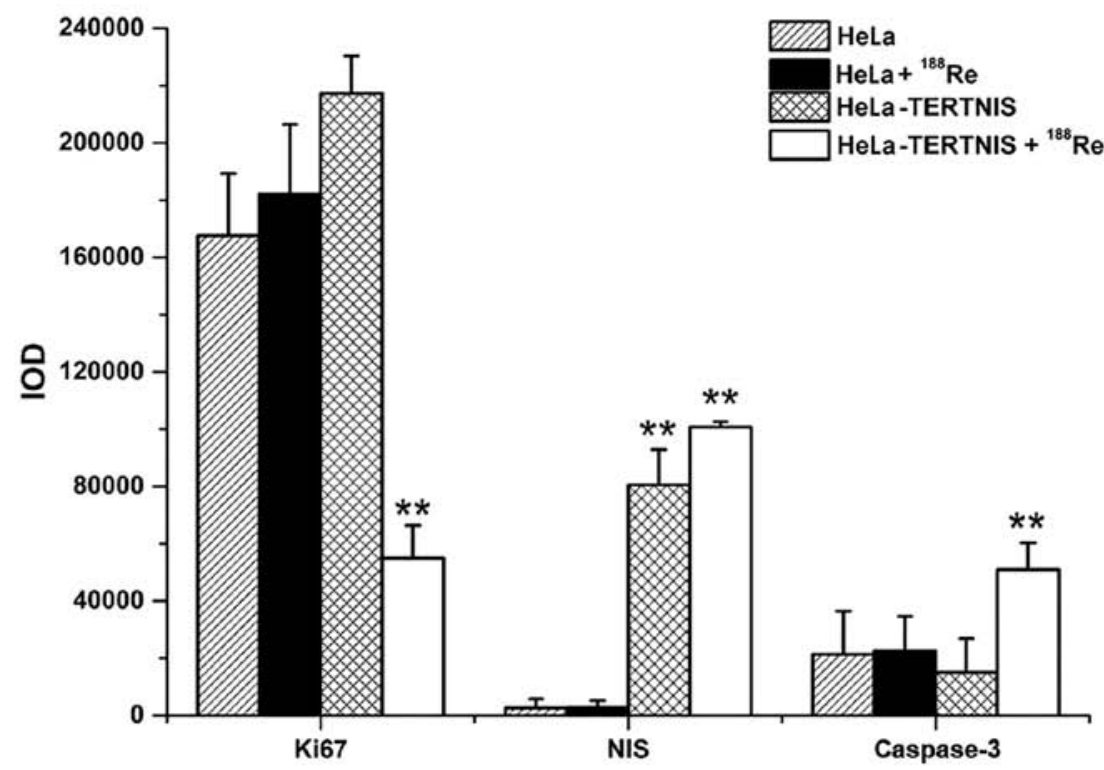

HeLa-TERTNIS HeLa-TERTNIS + ${ }^{188}$ Re
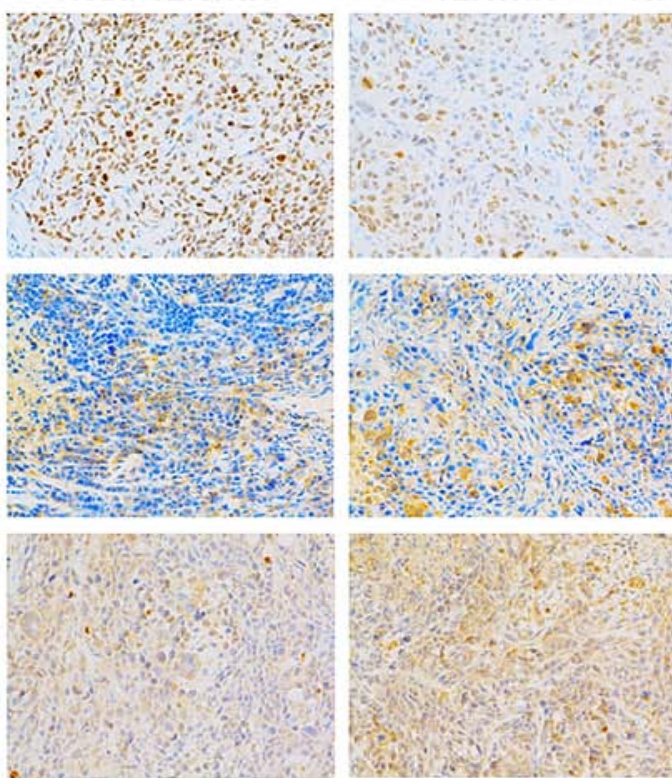

Ki67

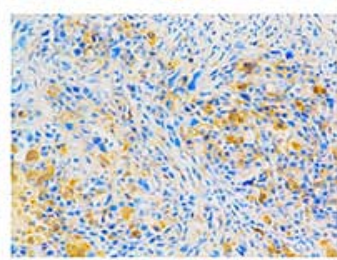

NIS

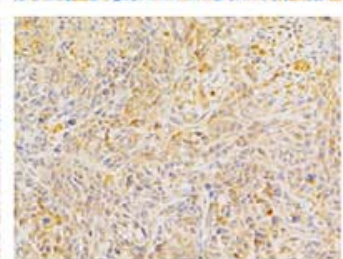

Caspase-3

Figure 8. Immunohistochemical staining. (A) Expression of NIS, caspase-3, and Ki-67 in HeLa and HeLa-TERTNIS xenograft tumors with or without ${ }^{188} \mathrm{Re}$ treatment (magnification, $\mathrm{x} 200$ ). Brown indicates positive staining. (B) Semi-quantitatively analysis of NIS, caspase-3, and Ki67 proteins on the basis of integral optical density (IOD). Data are present as means \pm SD. ${ }^{* *} \mathrm{P}<0.01$.

colon tumors, nasopharyngeal carcinomas, and lung adenocarcinoma (9-12) in our laboratory. However, a tumor-specific expression system for the NIS gene needs to be established in order to restrict the NIS-mediated uptake of radioisotopes to tumor cells and protect normal cells from the cytotoxic effects of radioisotopes.

Telomerase is almost undetectable in most normal somatic cells but is strongly expressed in embryonic and stem cells as well as in $85-90 \%$ cancer cells, in which it supports cell proliferation by maintaining telomeres. hTERT is the rate-limiting determinant of telomerase, and the hTERT promoter selectively promotes hTERT gene expression in tumor cells (5). Therefore, the hTERT promoter has been widely used to drive the expression of suicide and oncolytic genes such as those encoding caspase-8, Bax, thymidine kinase, and E1, resulting in cell apoptosis or lysis only in telomerase-positive tumor cells (13-16). In the present study, eGFP expression under the control of the hTERT promoter was significantly higher in the four cancer cell lines than in the normal MRC-5 and HK-2 cell lines, indicating that selective transcriptional activation by the hTERT promoter in cancer cells is an effective strategy for achieving the tumor-specific expression of transgenes. Of note, the transcription efficiency of tumor-specific promoters is usually weaker than that of commonly used promoters such as the cytomegalovirus and the simian virus 40 promoter, but NIS gene expression under the control of the hTERT promoter in the HeLa-TERTNIS cells was high in the present study. This suggested the unusually high transcription efficiency of the hTERT promoter, which was almost equal to that of the cytomegalovirus promoter described previously (7). Reportedly, 
the transcription efficiency of the hTERT promoter is even higher than that of the simian virus 40 promoter in human prostate cancer cell DU145 and human malignant melanoma SK-MEL-5 cells (13), indicating that the transcription efficiency of the hTERT promoter differed considerably among various cancer cell lines.

Therapeutic effectiveness depends not only on the level of NIS expression but also on the dose and retention time of the radioisotope in the tumor expressing NIS. ${ }^{131} \mathrm{I}$ $\left(\mathrm{E}_{\text {average }}=0.134 \mathrm{MeV}\right.$; physical half-life $=8.1$ days $)$ is the most frequently used radioisotope for NIS-based tumor therapy, but because of the lack of organification of iodide in non-thyroid cancers, it is not retained for an adequate time owing to its rapid efflux despite sufficient iodide uptake (17). Several approaches have been proposed to overcome this problem, one of which is the use of more powerful $\beta$-emitting radioisotopes transported by NIS, such as ${ }^{188} \operatorname{Re}\left(\mathrm{E}_{\text {average }}=0.764 \mathrm{MeV}\right.$; physical half-life $=16.7 \mathrm{~h}$ ). In our in vivo experiments, ${ }^{188} \mathrm{Re}$ uptake in HeLa-TERTNIS xenografts reached the maximal level at $0.5 \mathrm{~h}$ after injection and then decreased more rapidly than ${ }^{125} \mathrm{I}$ levels did, especially at the $24 \mathrm{~h}$ point. The shorter retention time of ${ }^{188} \mathrm{Re}$ than ${ }^{125} \mathrm{I}$ in the HeLa-TERTNIS xenografts might be a result of its faster clearance from circulation. Because of the thyroid gland reservoir and entero-recirculation of iodide, ${ }^{125}$ I clearance from circulation may be restricted. Despite the shorter retention time of ${ }^{188} \mathrm{Re}$, a previous study found that the ${ }^{188} \mathrm{Re}$ to ${ }^{131} \mathrm{I}$ ratio of tumor-absorbed dose at the same level of radioactivity was about 4.5:1 in a murine xenograft model for breast cancer (6). Encouraging results regarding ${ }^{188} \mathrm{Re}$ therapy have also been reported for NIS-transfected hepatocarcinoma (18) and glioma (19).

In the present study, ${ }^{188} \mathrm{Re}$ efficiently and specifically inhibited the growth of HeLa-TERTNIS tumor cells both in vitro and in vivo. Further, immunohistochemical analysis for Ki67 and caspase-3 showed a significant decrease in cell proliferation and increased levels of apoptosis after ${ }^{188} \mathrm{Re}$ treatment of HeLa-TERTNIS tumors. ${ }^{188} \mathrm{Re}$ also has the advantage of emitting $155 \mathrm{keV} \gamma$-rays, an energy comparable to that of ${ }^{99 \mathrm{~m}} \mathrm{Tc}$, whereby it enables SPECT imaging for biokinetics evaluation. Collectively, the results suggest that ${ }^{188} \mathrm{Re}$ is a suitable alternative for treatment of NIS-expressing non-thyroid tumors.

Gene therapy depends on transfer vectors that facilitate the expression of a therapeutic gene. Viral vectors such as adenovirus, adeno-associated virus, retrovirus, and baculovirus have been widely employed for gene therapy in animal studies and clinical trials (20). In addition to possessing the useful features of previously developed retroviral vectors, the lentiviral vector has the ability to transfect dividing and non-dividing cells and can harbor large target gene fragments, and recipients are unlikely to have pre-existing immunity to this virus (21). In the present study, the lentiviral vector facilitated strong and stable expression of NIS, which in turn ensured high levels of ${ }^{188} \mathrm{Re}$ or ${ }^{125} \mathrm{I}$ uptake in HeLa-TERTNIS xenografts. Our findings are superior to those concerning baculoviral vector-mediated transfer of the NIS gene obtained in a previous study (7) because of the higher transfection efficiency of lentivirus than baculovirus for mammalian cells. Although a potentially dangerous situation may arise with the use of the lentiviral vector, that is, the transgene may integrate into the genomic
DNA of host cells, the lentiviral vector remains a very efficient tool for NIS gene transfer.

In conclusion, a lentiviral vector containing the NIS gene driven by the hTERT promoter enabled efficient ${ }^{188} \mathrm{Re}$ uptake into cervical cancer HeLa cells and showed a significant therapeutic effect both in vitro and in vivo. Further, it enabled in vivo imaging of tumors. Our findings indicate the possibility of tumor-specific NIS gene therapy and imaging using the powerful radioisotope ${ }^{188} \mathrm{Re}$.

\section{Acknowledgements}

This work was supported by grants from the National Natural Science Foundation of China (NSFC) (81071181), and Shanghai Natural Science Foundation (13ZR1426000). We are indebted to the staff of the Department of Nuclear Medicine, Fudan University Shanghai Cancer Center for their technological support with micro-SPECT/CT imaging.

\section{References}

1. Torre LA, Bray F, Siegel RL, Ferlay J, Lortet-Tieulent J and Jemal A: Global cancer statistics, 2012. CA Cancer J Clin 65: 87-108, 2015

2. Koh WJ, Greer BE, Abu-Rustum NR, Apte SM, Campos SM, Cho KR, Chu C, Cohn D, Crispens MA, Dorigo O, et al: Cervical Cancer, Version 2.2015. J Natl Compr Canc Netw 13: 395-404, quiz 404, 2015.

3. Dai G, Levy O and Carrasco N: Cloning and characterization of the thyroid iodide transporter. Nature 379: 458-460, 1996.

4. Riesco-Eizaguirre G and Santisteban P: A perspective view of sodium iodide symporter research and its clinical implications. Eur J Endocrinol 155: 495-512, 2006.

5. Kim NW, Piatyszek MA, Prowse KR, Harley CB, West MD, Ho PL, Coviello GM, Wright WE, Weinrich SL and Shay JW: Specific association of human telomerase activity with immortal cells and cancer. Science 266: 2011-2015, 1994.

6. Dadachova E, Bouzahzah B, Zuckier LS and Pestell RG: Rhenium-188 as an alternative to Iodine-131 for treatment of breast tumors expressing the sodium/iodide symporter (NIS). Nucl Med Biol 29: 13-18, 2002.

7. Zhang M, Guo R, Shi S, Miao Y, Zhang Y and Li B: Baculovirus vector-mediated transfer of sodium iodide symporter and plasminogen kringle 5 genes for tumor radioiodide therapy. PLoS One 9: e92326, 2014.

8. Livak KJ and Schmittgen TD: Analysis of relative gene expression data using real-time quantitative PCR and the 2(-Delta Delta C(T)) method. Methods 25: 402-408, 2001.

9. Guo R, Zhang M, Xi Y, Ma Y, Liang S, Shi S, Miao Y and Li B: Theranostic studies of human sodium iodide symporter imaging and therapy using ${ }^{188} \mathrm{Re}$ : A human glioma study in mice. PLoS One 9: e102011, 2014.

10. Shi S, Zhang M, Guo R, Miao Y, Hu J, Xi Y and Li B: In vivo molecular imaging and radionuclide (131I) therapy of human nasopharyngeal carcinoma cells transfected with a lentivirus expressing sodium iodide symporter. PLoS One 10: e0116531, 2015.

11. Yin HY, Zhou X, Wu HF, Li B and Zhang YF: Baculovirus vector-mediated transfer of NIS gene into colon tumor cells for radionuclide therapy. World J Gastroenterol 16: 5367-5374, 2010.

12. Guo R, Zhang Y, Liang S, Xu H, Zhang M and Li B: Sodium butyrate enhances the expression of baculovirus-mediated sodium/iodide symporter gene in A549 lung adenocarcinoma cells. Nucl Med Commun 31: 916-921, 2010.

13. Koga S, Hirohata S, Kondo Y, Komata T, Takakura M, Inoue M, Kyo S and Kondo S: A novel telomerase-specific gene therapy: Gene transfer of caspase-8 utilizing the human telomerase catalytic subunit gene promoter. Hum Gene Ther 11: 1397-1406, 2000.

14. Gu J, Andreeff M, Roth JA and Fang B: hTERT promoter induces tumor-specific Bax gene expression and cell killing in syngenic mouse tumor model and prevents systemic toxicity. Gene Ther 9: $30-37,2002$. 
15. Fujiwara T, Urata Y and Tanaka N: Telomerase-specific oncolytic virotherapy for human cancer with the hTERT promoter. Curr Cancer Drug Targets 7: 191-201, 2007.

16. Yu B, Zhang Y, Zhan Y, Zha X, Wu Y, Zhang X, Dong Q, Kong W and Yu X: Co-expression of herpes simplex virus thymidine kinase and Escherichia coli nitroreductase by an hTERT-driven adenovirus vector in breast cancer cells results in additive antitumor effects. Oncol Rep 26: 255-264, 2011.

17. Haberkorn U, Kinscherf R, Kissel M, Kübler W, Mahmut M, Sieger S, Eisenhut M, Peschke P and Altmann A: Enhanced iodide transport after transfer of the human sodium iodide symporter gene is associated with lack of retention and low absorbed dose. Gene Ther 10: 774-780, 2003.

18. Kang JH, Chung JK, Lee YJ, Shin JH, Jeong JM, Lee DS and Lee MC: Establishment of a human hepatocellular carcinoma cell line highly expressing sodium iodide symporter for radionuclide gene therapy. J Nucl Med 45: 1571-1576, 2004.
19. Shen DH, Marsee DK, Schaap J, Yang W, Cho JY, Hinkle G, Nagaraja HN, Kloos RT, Barth RF and Jhiang SM: Effects of dose, intervention time, and radionuclide on sodium iodide symporter (NIS)-targeted radionuclide therapy. Gene Ther 11: 161-169, 2004.

20. Kohn DB and Candotti F: Gene therapy fulfilling its promise. N Engl J Med 360: 518-521, 2009.

21. Vigna E and Naldini L: Lentiviral vectors: Excellent tools for experimental gene transfer and promising candidates for gene therapy. J Gene Med 2: 308-316, 2000. 